\title{
Generating nonlinearities from conditional linear operations, squeezing, and measurement for quantum computation and super-Heisenberg sensing
}

\author{
Mattias T. Johnsson $\odot,{ }^{1}$ Pablo M. Poggi $\odot,{ }^{2}$ Marco A. Rodriguez $\odot,{ }^{3}$ Rafael N. Alexander,,${ }^{2,4}$ and Jason Twamley ${ }^{5,1, *}$ \\ ${ }^{1}$ Centre for Engineered Quantum Systems, Department of Physics and Astronomy, \\ Macquarie University, Sydney, New South Wales 2109, Australia \\ ${ }^{2}$ Center for Quantum Information and Control, University of New Mexico, MSC07-4220, Albuquerque, New Mexico 87131-0001, USA \\ ${ }^{3}$ Instituto de Investigaciones en Matematicas Aplicadas y Sistemas, Universidad Nacional Auntonoma de Mexico, CDMX. 01000, Mexico \\ ${ }^{4}$ Centre for Quantum Computation and Communication Technology, School of Science, RMIT University, Melbourne, Victoria 3001, Australia \\ ${ }^{5}$ Quantum Machines Unit, Okinawa Institute of Science and Technology Graduate University, Okinawa 904-0495, Japan
}

(Received 24 December 2020; accepted 17 May 2021; published 21 June 2021; corrected 22 July 2021)

\begin{abstract}
Large bosonic or continuous variable nonlinearities can have numerous applications, ranging from the generation of cat states for quantum computation, through to quantum sensing where the sensitivity exceeds Heisenberg scaling in the resources. However, the generation of ultra-large nonlinearities has proved immensely challenging experimentally. We describe a novel protocol where one can effectively generate large Kerr-type nonlinearities via the conditional application of a linear operation on an optical mode by an ancilla mode, followed by a measurement of the ancilla and corrective operation on the probe mode. Our protocol can generate high-quality Schrödinger cat states useful for quantum computing and can be used to perform sensing of an unknown rotation or displacement in phase space, with super-Heisenberg scaling in the resources. We finally describe a potential experimental implementation using atomic ensembles interacting with optical modes via the Faraday effect.
\end{abstract}

DOI: 10.1103/PhysRevResearch.3.023222

\section{INTRODUCTION}

Optical nonlinearities, and particularly the Kerr nonlinear oscillator, have been the focus of much research within quantum optics since the investigations by Milburn and Holmes [1] and Yurke and Stoler [2]. Nonlinear quantum oscillators and the highly nonclassical cat states they can produce have found numerous applications including studying the fundamentals of decoherence [3], improved schemes for metrology [4-28], as well as for quantum computation [29-31]. Researchers have shown that nonlinear quantum systems can provide a metrological precision that scales better than the so-called Heisenberg scaling in the estimation of a parameter $\phi$. Standard quantum limit (SQL) and Heisenberg quantum limit (HL) metrology schemes result in an imprecision $\delta \phi$ that scales with the resource $\bar{n}$ as $\delta \phi \sim 1 / \sqrt{\bar{n}}$ or $1 / \bar{n}$, respectively.

Although nonlinear Kerr-type oscillators have been intensively studied theoretically, experimentally implementing them has proved extremely challenging. The degree of nonlinearity that can be engineered in most atomic or optical systems is too small or is associated with too much loss to be useful. Recently, superconducting quantum devices have proved capable of generating Kerr-type quantum states in the

\footnotetext{
*jason.twamley1@ oist.jp

Published by the American Physical Society under the terms of the Creative Commons Attribution 4.0 International license. Further distribution of this work must maintain attribution to the author(s) and the published article's title, journal citation, and DOI.
}

microwave domain [32], but their generation in the optical domain remains problematic.

In this paper, we show that, curiously, one can imprint a nonlinear Hamiltonian (in our case a Kerr) on an optical mode (which we will denote as the probe mode) using only a conditional linear operation from an ancilla mode which is then measured. This measurement implements a Kraus operation on the primary mode which comprises both unitary and nonunitary components, and in a suitable limit, we find that the Kraus operation is almost of a pure Kerr type. We show how tailoring this Kraus operation

(1) can be used to perform super-Heisenberg sensing of an unknown rotation in phase space with an imprecision which scales as $\delta \theta \sim 1 / \bar{n}^{3 / 2}$, where $\bar{n}$ is the mean Fock number of the probe mode;

(2) can be used to perform super-Heisenberg sensing of an unknown displacement in phase space with $\delta x \sim 1 / \bar{n}^{3 / 2}$;

(3) can be used to engineer near perfect nonclassical Kerr cats for use in optical quantum computation and metrology; and

(4) can be implemented using optical modes interacting with atomic ensembles via the Faraday effect.

In this paper, we consider the coupling of an optical probe mode to an ancillary pseudobosonic degree of freedom that arises from a collection of spins as a system that couples to optical mode. We assume access to a fixed but large degree of spin squeezing in this system. In Sec. II, we describe the fundamental idea behind the scheme, which is graphically depicted in Fig. 1. In Sec. III, we introduce the reader to some principles of quantum metrology, and in Sec. III A, we give a quick introduction to quantum Fisher information (QFI). 


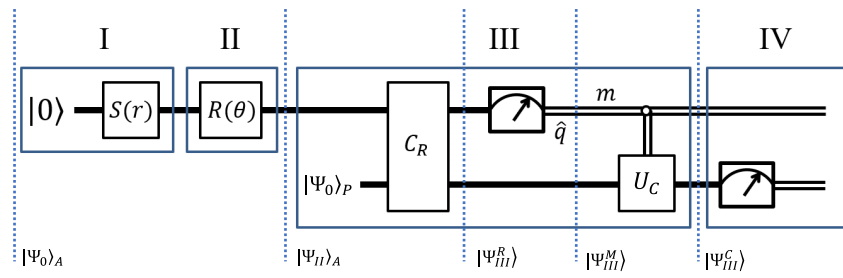

FIG. 1. Basic protocol: Both the top and bottom lines of the circuit represent bosonic modes, which we label as the ancilla (top) and probe (bottom) modes. In Stage I, we initialize the ancilla mode in a squeezed vacuum state, where $S(r)$ is the squeezing operator. In Stage II, we implement a rotation of the ancilla mode by $R(\theta)$. In Stage III, we implement "Kerr teleportation" onto the input state $|\psi\rangle$ (which we assume is a coherent state), via the cross-rotation gate $C_{R}$ (which rotates the probe mode by an amount dependent on the momentum of the ancilla mode, e.g., generated by $\hat{H}_{R}=\tilde{g} \hat{p}_{A} \otimes \hat{n}_{P}$ ), and a position quadrature measurement of the first mode. This has the upshot of implementing both linear $\sim \hat{n}_{P}$ and quadratic $\sim \chi \hat{n}_{P}^{2}$ operations on the probe mode, where the Kerr strength $\chi$ is a function of $r, \theta$, and $g$. We allow for a measurement outcome dependent unitary $U_{c}$ at the conclusion of this teleportation procedure to undo the linear rotations, leaving only Kerr-like evolution with some decay. Finally, the probe mode can be measured in Stage IV. Quantum states referred to in the text at various points in the circuit are labeled by dotted vertical lines.

In Sec. III B, we extend the QFI to postmeasurement ensembles, and in Secs. III C and III D, we describe how to perform super-Heisenberg metrology. In Sec. IV, we describe how to use our protocol to generate Schrödinger cat and compass states with high fidelity, and finally, in Sec. V, we describe a potential experimental implementation of our protocol which uses collective modes of an atomic ensemble in the HolsteinPrimakoff approximation as an effective harmonic oscillator mode.

\section{OUTLINE OF OUR PROTOCOL TO GENERATE NONLINEAR DYNAMICS}

The protocols we develop in this paper are primarily based on the innocuous Gaussian integral

$$
\int_{-\infty}^{+\infty} d y \exp \left(-a y^{2}+b y\right)=\sqrt{\frac{\pi}{a}} \exp \left(\frac{b^{2}}{4 a}\right),
$$

which converges provided $\operatorname{Re}(a)>0$. This integration has the interesting property that the parameter $b$, which appears linearly in the exponential of the integrand on the left, ends up appearing quadratically in the exponential on the right. Unitary operators can be written as the exponential of an Hermitian generator. We will make use of the curious property of Eq. (1) to essentially square the generator. We will show how it is possible to bootstrap the typical harmonic oscillator generator $\hat{H}_{\mathrm{ho}} \sim \hat{a}^{\dagger} \hat{a} \sim \hat{n}$ to become that of the nonlinear Kerr oscillator $\hat{H}_{\mathrm{ko}} \sim \hat{n}^{2}$. We will see that this bootstrapping can only be achieved approximately with realistic resources, but interestingly, it can be achieved deterministically even though a measurement is involved.

We introduce this protocol via Fig. 1, which is the basic description of the bootstrap protocol. We begin with two bosonic modes, as shown in Fig. 1, with the top (bottom) mode denoted as the ancilla (probe) modes. We introduce the following single- and two-mode operators:

$$
\begin{aligned}
\hat{S}(r) & =\exp \left[\frac{-r\left(\hat{a}_{A}^{2}-\hat{a}_{A}^{\dagger 2}\right)}{2}\right], \\
\hat{R}(\theta) & =\exp \left(i \theta \hat{n}_{A}\right), \\
\hat{S h}(\beta) & =\exp \left(-i \beta \hat{p}_{A}^{2}\right), \\
\hat{C}_{R} & =\exp \left(-i g \hat{p}_{A} \otimes \hat{n}_{P}\right),
\end{aligned}
$$

where the $A(P)$ subscript indicates operators acting on the ancilla (probe) modes. These operators we name as the $S(r)$ : squeezing, $R(\theta)$ : rotation, $S h(\beta)$ : shear, and $C_{R}$ : cross-rotation operators. Referring to Fig. 1, we begin by considering Stages I and II involving the ancilla mode where, for simplicity, initially, we will apply $\hat{S h}(\beta)$ at Stage II rather than $\hat{R}(\theta)$. We will generalize to the case including rotation later. We can write the state of the ancilla mode after Stage II as

$$
\begin{aligned}
\left|\psi_{I I}\right\rangle_{A} & \equiv \hat{S h}(\beta) \hat{S}(r)|0\rangle_{A} \\
& =\exp \left(-i \beta \hat{p}_{A}^{2}\right) \hat{S}(r)|0\rangle_{A} \\
& =\mathcal{N}_{I I} \int d p \exp \left(-\frac{p^{2}}{2 \sigma^{2}}\right) \exp \left(-i \beta p^{2}\right)|p\rangle_{A}
\end{aligned}
$$

where $|p\rangle_{A}$ is the eigenstate of $\hat{p}_{A}$, the momentum operator of the ancilla mode, $\sigma^{2}=e^{2 r}$, and $\mathcal{N}_{I I}$ is a normalization constant. We next bring in the initial state of the probe mode $|\psi\rangle_{P} \equiv|\alpha\rangle_{P}$, taking it to be in a coherent state of magnitude $\alpha$, and apply the cross-rotation gate to obtain

$$
\begin{aligned}
\left|\Psi_{I I I}^{R}\right\rangle \equiv & \exp \left(-i g \hat{p}_{A} \otimes \hat{n}_{P}\right)\left|\psi_{I I}\right\rangle_{A} \otimes|\alpha\rangle_{P} \\
= & \mathcal{N}_{I I} \int d p \exp \left[-p^{2}\left(\frac{1}{2 \sigma^{2}}+i \beta\right)\right] \\
& \times \exp \left(-i g p \hat{n}_{P}\right)|p\rangle_{A} \otimes|\alpha\rangle_{P} .
\end{aligned}
$$

Next, in Stage III, we apply a position measurement on the ancilla mode, and if the result of that measurement is $m$, and given $\langle m \mid p\rangle_{A}=e^{\mathrm{imp}} / \sqrt{2 \pi}$, we obtain the postmeasured state of the probe to be

$$
\begin{aligned}
\left|\Psi_{I I I}^{M}\right\rangle= & \mathcal{N}_{I I I M} \int d p \exp \left[-p^{2}\left(\frac{1}{2 \sigma^{2}}+i \beta\right)\right] \\
& \times \exp \left[i p\left(m-g \hat{n}_{P}\right)\right]|\alpha\rangle_{P},
\end{aligned}
$$

where $\mathcal{N}_{I I I M}=1 /\left(\pi^{1 / 4} \sqrt{2 \pi \sigma}\right)$. We can now proceed to integrate over the integration variable $p$. For this, we use the known integral

$$
\int_{-\infty}^{+\infty} d p \exp \left(-a p^{2}+b p\right)=\sqrt{\frac{\pi}{a}} \exp \left(\frac{b^{2}}{4 a}\right),
$$

which holds only if $\operatorname{Re}(a)>0$. Identifying $a=1 / 2 \sigma^{2}+i \beta$ and $b=i\left(m-g \hat{n}_{P}\right)$, we obtain for the postmeasurement state of the probe mode

$$
\begin{aligned}
\left|\Psi_{I I I}^{M}\right\rangle & =\mathcal{N}_{I I I M}^{\prime} \exp \left[-\frac{(g \hat{n}-m)^{2}}{4\left(\frac{1}{2 \sigma^{2}}+i \beta\right)}\right]|\alpha\rangle \\
& =\mathcal{N} \hat{U}(\beta) \hat{U}_{c}(\beta, m) \hat{K}(\beta) \hat{K}_{c}(\beta, m)|\alpha\rangle,
\end{aligned}
$$


where $\mathcal{N}$ is a normalization factor, and we have now dropped the subscript $P$ referring to the probe mode. In the final expression Eq. (14), we have decomposed the propagator into a product of four commuting operators which describe the complete action of the channel on the initial state of the probe. These include two unitary operations $\hat{U}$ and $\hat{U}_{c}$ and two nonunitary operations $\hat{K}$ and $\hat{K}_{c}$. Their explicit form is given by

$$
\begin{aligned}
\hat{U}(\beta) & =\exp \left(i \frac{\beta g^{2}}{\mu} \hat{n}^{2}\right), \\
\hat{K}(\beta) & =\exp \left(-\frac{g^{2}}{2 \sigma^{2} \mu} \hat{n}^{2}\right), \\
\hat{U}_{c}(\beta, m) & =\exp \left(i \frac{2 \beta m g}{\mu} \hat{n}\right), \\
\hat{K}_{c}(\beta, m) & =\exp \left(-\frac{2 m g}{2 \sigma^{2} \mu} \hat{n}\right),
\end{aligned}
$$

where $\mu=4\left[\beta^{2}+1 /\left(4 \sigma^{4}\right)\right]$. We notice that the evolution of the probe state involves deterministic operations $\hat{U}(\beta)$ and $\hat{K}(\beta)$, which do not depend on the measurement result $m$, as well as conditioned operations $\hat{U}_{c}(\beta, m)$ and $\hat{K}_{c}(\beta, m)$, which do depend on $m$.

We now reach our first important observation: the unitary $\hat{U}(\beta)$ is a deterministic pure Kerr evolution, where the Kerr strength $\chi \equiv \beta g^{2} / \mu$ now depends on $\beta$ and $\sigma$, the shear and squeezing parameters of the operations on the ancilla mode. We also see that all the operations in Eqs. (15)-(18) commute irrespective of the parameter values. In the limit of infinite squeezing $\sigma \rightarrow \infty$, we have $\mu \rightarrow 4 \beta^{2}$, and the two nonunitary operators collapse to the identity, while $\chi \rightarrow g^{2} / 4 \beta$, and $\hat{U}_{c}$ is an $m$-dependent rotation with phase $\phi=m g / 2 \beta$. In this limit, we are left with pure unitary evolution that consists of a deterministic nonlinear rotation and a measurementdependent linear rotation. In the final rightmost section of III in Fig. 1, we assume we can apply a correction unitary on the probe mode which depends on the value of the measurement outcome $m$.

In the above simplified initial description of the protocol, we applied the shearing operator $\hat{S h}(\beta)$, rather than a rotation operator $\hat{R}(\theta)$. Use of the shearing operation permits a relatively straightforward illustration of the main principles of how the protocol operates, and the resulting analytic expressions are compact. However, it is more physically relevant to use the rotation operator $\hat{R}$. We note that the combination of squeeze and shear operations can be decomposed into separate squeeze and rotation operations and this alternative parametrization is shown in Fig. 1. We will make use of this latter description in the remainder of the paper below. To find this alternative parametrization, we use the Siegel upper half space representation of Gaussian pure states, where the state is represented by a complex number $z=v+i u$, where $u>0$ [33]. We note that the symplectic matrices corresponding to squeezing and rotation can be written as

$$
\left(\begin{array}{cc}
0 & -1 \\
1 & 0
\end{array}\right)\left(\begin{array}{cc}
\cos \theta & -\sin \theta \\
\sin \theta & \cos \theta
\end{array}\right)\left(\begin{array}{cc}
e^{-r} & 0 \\
0 & e^{r}
\end{array}\right)=\left(\begin{array}{ll}
a & b \\
c & d
\end{array}\right),
$$

where the leftmost matrix implements an extra $\pi / 2$ rotation which is responsible for interchanging the roles of the position and momentum bases, and the state gets squeezed in position for $r>0$. Then

$$
\frac{c+i d}{a+i b}=z=v+i u
$$

where $u=1 / \Xi, v=-\sin 2 \theta \sinh 2 r / \Xi$, and $\Xi=e^{-2 r} \sin ^{2} \theta+$ $e^{2 r} \cos ^{2} \theta$.

One can express the state output from Stage II as

$$
\begin{aligned}
\left|\psi_{I I}\right\rangle_{A} & =\hat{R}(\theta) \hat{S}(r)|0\rangle \\
& =\left(\frac{u}{\pi}\right)^{1 / 4} \int d p \exp \left[-\frac{1}{2} p^{2}(u-i v)\right]|p\rangle_{A},
\end{aligned}
$$

and repeating the steps from Eqs. (8)-(14), we can show

$$
\begin{aligned}
\frac{1}{2 \sigma^{2}} & =u, \\
\beta & =-v,
\end{aligned}
$$

and using Eqs. (23) and (24), one can re-express the parameters $(\sigma, \beta) \rightarrow(r, \theta)$. We will primarily use the $(r, \theta)$ parametrization in the remainder of the paper below.

In the remainder of the paper, we will explore two main variations of this circuit:

Enhanced quantum metrology: If we assume we have imperfect knowledge of one parameter in the circuit, e.g., of the angle $\theta$, and wish to estimate the value of $\theta$, we first describe how, using the circuit in Fig. 1, we can perform this estimate with a precision that scales as $\Delta \theta \sim 1 / \bar{n}_{P}^{3 / 2}$, where $\bar{n}_{P}$ is the mean Fock number of the input probe coherent state. This scaling in precision is faster than the typical Heisenberg scaling for estimating $\theta$, which normally scales as $\Delta \theta \sim 1 / \bar{n}_{P}$. The ancilla mode can have high levels of squeezing, but in the protocol, we consider the resources of the ancilla mode to be fixed, while we vary the Fock number $\bar{n}_{P}$ of the probe mode. We describe how the circuit shown in Fig. 1 can be used to estimate $\theta$, the parameter of a phase rotation of a mode, and alternatively, how to estimate $\kappa$, which parameterizes displacements of a mode, each with a precision that scales as $\sim 1 / \bar{n}_{P}^{3 / 2}$. We note that, in this latter example, which is often used for force sensing, the standard HL scales as $\Delta \kappa \sim 1 / \sqrt{\bar{n}}$. Thus, our improvement in displacement metrology over the normal HL is substantial [34]. We discuss this scaling further in Sec. III D.

Non-Gaussian state preparation: Next, we will assume we have full information of all the parameters in the circuit. With full knowledge of these parameters, we can apply complete nonlinear correction so that, in the high-squeezing limit, we are left with a deterministic pure Kerr evolution $\hat{U}(\theta)=\exp \left[i\left(g^{2} / 4\right) \cot (\theta) \hat{n}^{2}\right]$, which, curiously, has a Kerr strength which is a highly nonlinear function of $\theta$. We can use this to produce nonclassical states of the probe mode and, with infinite squeezing and pure-Kerr evolution, we can target the generation of a Yurke-Stoler cat state [2]. A more realistic scenario, using finite squeezing, will result in imperfect preparation of such non-Gaussian quantum states. In Sec. IV, we study the preparation fidelities that can be achieved using this scheme. 


\section{PERFORMING ENHANCED METROLOGY}

We now develop the quantum circuit outlined in Fig. 1 to perform parameter estimation as outlined at the end of Sec. II. We assume that we have complete knowledge of the parameters $\left(r, g, m, \alpha_{P}\right)$ denoting the ancilla mode squeezing, strength of the cross-rotation gate $C_{R}$, measurement outcome $m$, and the parameter $\alpha_{P}$ describing the coherent-state input to the probe mode, respectively. We assume that the parameter $\theta$ is set to a particular known base value (which we denote as $\theta$ ), and we are interested in estimating changes in $\theta \rightarrow \theta+\delta \theta$ about this base value with precision. We also assume we can apply a correction rotation unitary at the end of Stage III of the circuit shown in Fig. 1, or $\hat{U}_{c}[f(r, g, \theta), m]$ in Eq. (17), where $f$ is a deterministic function of these known parameters. This correction unitary aims to reduce the stochastic effects of the measurement on the probe. To gain some intuition regarding the necessity of this correction unitary, we refer the reader to its use in the quantum teleportation of qubits. In teleportation, Alice performs a joint measurement on a qubit in an unknown state and also on one half of an entangled pair of qubits, while the other qubit of this entangled pair is sent to Bob. If Alice sends her measurement result to Bob, the teleportation protocol can only be successful once Bob applies a correction unitary to his qubit given Alice's result. If Alice chooses not to tell Bob her result, then Bob's qubit is left in a complete mixture. We uncover a very similar situation below. If we choose not to apply a correction unitary, the unconditioned (averaged over measurements) state of the probe mode is left in an extremely mixed state and retains little information useful for metrology. Once the appropriate unitary correction is applied, the unconditioned probe mode is much purer and retains significant amounts of information regarding the unknown parameter which can be extracted via a final measurement on the probe mode. This final measurement on the probe mode is made to estimate the unknown parameter change $\delta \theta$. As a measure of the resources required to achieve a particular precision in estimation, we will make use of the QFI. The QFI is typically defined for unitary channels, where the effect on the final state by a change in the parameter is unitary. However, in the circuit shown in Fig. 1, information about $\delta \theta$ is found not only in the final conditioned state of the probe mode $\hat{\rho}_{P}^{m}$, but also in the classical measurement results $m$. We have to then expand the normal unitary QFI to encompass the ensemble of joint classical-quantum output states $\left\{m, \hat{\rho}_{P}^{m}\right\}$.

\section{A. Quick review of QFI}

In quantum metrology, one aims to statistically estimate the value of a parameter in the system using an unbiased estimator. From the quantum Cramér-Rao theorem, the QFI provides a lower limit on the variance of such an estimator [35-39]. A larger value of the QFI implies higher precision parameter estimation, and one can study the dependence of the QFI on various quantum resources, e.g., the average Fock number of the input probe mode. We now give the reader a quick overview of the properties of the QFI illustrated with a number of examples before addressing the QFI of a channel of pure states conditioned on classical measurement outcomes.
As mentioned above, the precision of a statistical estimation of a parameter $\theta$ can be studied in terms of the (classical) Fisher information (FI) $F(\theta)$, which determines the CramérRao bound for the variance of an unbiased estimator

$$
\Delta \theta \geqslant \Delta \theta_{\mathrm{CR}}=\frac{1}{\sqrt{v F(\theta)}},
$$

where $v$ quantifies the total number of repetitions of the estimation.

The FI can be upper bounded by the QFI $F_{Q}$. The QFI is a function of a family of parameterized quantum states $\{\hat{\rho}(\theta)\}$

$$
F_{Q}[\hat{\rho}(\theta)]=\operatorname{Tr}\left[\hat{\rho}(\theta) \hat{L}^{2}\right]
$$

where

$$
\frac{\partial \hat{\rho}(\theta)}{\partial \theta} \equiv \frac{1}{2}[\hat{\rho}(\theta) \hat{L}+\hat{L} \hat{\rho}(\theta)]
$$

and where $\hat{L}$ is a $\theta$-dependent Hermitian operator called the symmetric logarithmic derivative (SLD). When $\hat{\rho}(\theta)$ is pure, the SLD and the QFI are easy to calculate (see Refs. [40,41]). Because $\rho_{\theta}=\rho_{\theta}^{2}$, we have

$$
\frac{\partial \hat{\rho}(\theta)}{\partial \theta}=\frac{\partial}{\partial \theta}\left[\hat{\rho}^{2}(\theta)\right]=\frac{\partial \hat{\rho}(\theta)}{\partial \theta} \hat{\rho}(\theta)+\hat{\rho}(\theta) \frac{\partial \hat{\rho}(\theta)}{\partial \theta},
$$

which immediately gives, from Eq. (26),

$$
\hat{L}=2 \frac{\partial \hat{\rho}(\theta)}{\partial \theta}=\left|\partial_{\theta} \psi_{\theta}\right\rangle\left\langle\psi_{\theta}|+| \psi_{\theta}\right\rangle\left\langle\partial_{\theta} \psi_{\theta}\right|,
$$

where we have expressed $\hat{\rho}(\theta)=\left|\psi_{\theta}\right\rangle\left\langle\psi_{\theta}\right|$ and denoted $\frac{\partial}{\partial \theta} \rightarrow \partial_{\theta}$. We can thus write the QFI for pure states

$$
F_{Q}[\hat{\rho}(\theta)]=\left\langle\psi_{\theta}\left|\hat{L}^{2}\right| \psi_{\theta}\right\rangle=4\left(\left\langle\partial_{\theta} \psi_{\theta} \mid \partial_{\theta} \psi_{\theta}\right\rangle-\left|\left\langle\psi_{\theta} \mid \partial_{\theta} \psi_{\theta}\right\rangle\right|^{2}\right) .
$$

If the parameterized pure states $\left\{\left|\psi_{\theta}\right\rangle\right\}$ are generated by a $\theta$-dependent unitary transformation acting on a fiducial state $\left|\psi_{0}\right\rangle$, i.e., if $\left|\psi_{\theta}\right\rangle=\exp (-i \hat{G} \theta)\left|\psi_{0}\right\rangle$, then the expression above reduces to

$$
F_{Q}\left[\rho_{\theta}\right]=F_{Q}\left[\left|\psi_{0}\right\rangle, \hat{G}\right]=4\left(\left\langle\psi_{0}\left|\hat{G}^{2}\right| \psi_{0}\right\rangle-\left\langle\psi_{0}|\hat{G}| \psi_{0}\right\rangle^{2}\right),
$$

which is four times the variance of the generator $\hat{G}$ in that fiducial state $\left|\psi_{0}\right\rangle$, which we will denote as $\left.(\Delta \hat{G})\right|_{\psi_{0}} ^{2}$.

For illustrative purposes, we now consider some applications of these QFI relations with respect to metrology. We first consider estimating an unknown phase imprinted on the state of a single quantum bosonic mode prepared in the fiducial coherent state $|\alpha\rangle$, which is subject to an unknown linear phase shift via the operation $R(\theta)=\mathrm{e}^{-i \theta \hat{n}}$, where $\hat{n}=\hat{a}^{\dagger} \hat{a}$. From Eq. (30), we find the pure state QFI as $F_{Q}[|\alpha\rangle, \hat{n}]=$ $\left.4(\Delta \hat{n})\right|_{\alpha} ^{2}=4|\alpha|^{2}=4 \bar{n}$, using the notation for the variance introduced above. Here, we have also introduced $\bar{n}$ as the mean number occupation of the fiducial state. We will be particularly focused on analyzing the scaling of the QFI with $\bar{n}$ for different types of metrology protocols, treating $\bar{n}$ as a quantification of the quantum resource. In this case, the scaling $F_{Q}[|\alpha\rangle, \hat{n}] \sim \bar{n}$, represents the SQL for phase estimation. The SQL for phase estimation can be beaten by imprinting linear phase shifts on squeezed states, as shown, for example, in Ref. [42]. An input fiducial state which is a squeezed 
vacuum state $\left|\phi_{r}\right\rangle=\hat{S}(r)|0\rangle$, with mean Fock number $\bar{n}$, yields a QFI $F_{Q}\left[\left|\phi_{r}\right\rangle, \hat{n}\right]=\left.4(\Delta \hat{n})\right|_{\phi_{r}} ^{2}=8 \sinh (r)^{2} \cosh (r)^{2}=$ $8\left(\bar{n}^{2}+\bar{n}\right)$, and thus leads to a better scaling of the QFI with $\bar{n}$, or the so-called Heisenberg scaling of the estimation of the phase, where $\Delta \theta \sim 1 / \bar{n}$.

Finally, we look at the case of generating a nonlinear phase shift on a coherent state, that is, applying a transformation like $\mathrm{e}^{-i \theta \hat{n}^{2}}$ to $|\alpha\rangle$. Following Ref. [13], it is not hard to show that

$$
F_{Q}\left[|\alpha\rangle, \hat{n}^{2}\right]=\left.4\left(\Delta \hat{n}^{2}\right)\right|_{\alpha} ^{2}=4\left(4 \bar{n}^{3}+6 \bar{n}^{2}+\bar{n}\right),
$$

and we observe a $\bar{n}^{3}$ scaling of the QFI. This results in super-Heisenberg scaling for the phase estimations, where $\Delta \theta \sim 1 / \bar{n}^{3 / 2}$, where $\bar{n}$ is the mean occupation of the probe mode. Experimentally, super-Heisenberg precision has only been demonstrated using a nonlinear atomic interferometer [43] and using many-body couplings in nuclear magnetic resonance [26]. Before describing how to achieve superHeisenberg scaling for phase estimation of an unknown phase $\theta$, using the protocol shown in Fig. 1, we first outline how the QFI generalizes to the outputs of the circuit shown in Fig. 1.

\section{B. QFI of a postmeasurement ensemble}

In the above, we considered the QFI associated with a parameter $\theta$, which modulates a unitary evolution of the initial fiducial state. In the scheme described in Fig. 1, however, the final state of a single run of the quantum circuit returns $\left[m(\theta), \hat{\rho}_{P}(m, \theta)\right]$, where $m$ is the classical measurement result, and $\hat{\rho}_{P}(m, \theta)$ is the corresponding conditional state of the probe mode associated with that measurement result. As the measurement results $m$ are random from run to run, the resulting average quantum evolution that the probe suffers is nonunitary, and we have to generalize the Cramer-Rao bound and QFI to this ensemble case. The appropriate formalism has been consider previously; see, for example, Refs. [44-46]. In what follows, we take the approach of Ma et al. [46], which we now briefly summarize.

We consider an extended system consisting of the system of interest (our probe mode) and an environment to which the system couples unitarily (our ancilla mode) and define the full density matrix of the extended system as $\hat{\rho}_{\text {ext }}(\theta)=\hat{U}_{\text {ext }}(\theta)\left(\left|E_{0}\right\rangle\left\langle E_{0}\right| \otimes \hat{\rho}_{0}\right) \hat{U}_{\text {ext }}^{\dagger}(\theta)$, where $\left|E_{0}\right\rangle$ is the $\theta$ independent initial state of the environment, and $\hat{\rho}_{0}$ is the initial state of the system. The environment is then traced out in a $\theta$-independent basis $\left\{\left|E_{l}\right\rangle\right\}$, and the reduced density matrix for the system alone can be written as

$$
\hat{\rho}_{\text {sys }}(\theta)=\operatorname{Tr}_{E} \hat{\rho}_{\text {ext }}(\theta)=\sum_{l} \hat{\Pi}_{l}(\theta) \hat{\rho}_{0} \hat{\Pi}_{l}^{\dagger}(\theta)=\sum_{l} \hat{\tilde{\rho}}_{l}(\theta),
$$

where $\Pi_{l}(\theta)=\left\langle E_{l}\left|U_{\text {ext }}(\theta)\right| E_{0}\right\rangle$ are Kraus operators operating on the system. Essentially, the $\hat{\tilde{\rho}}_{l}(\theta)$ are a set of quantum trajectories that occur with probability $P_{l}(\theta)=\operatorname{Tr}\left[\hat{\tilde{\rho}}_{l}(\theta)\right]$, and in our case, they will be pure states. In this picture, an optimal measurement of the quantum system to estimate $\theta$ yields the generalized QFI

$$
\mathbb{F}=F\left[\left\{P_{l}\right\}\right]+\sum_{l} P_{l} F_{Q}\left[\hat{\bar{\rho}}_{l}\right]
$$

where $F$ is the classical FI of the distribution of measurement results given by $F\left[\left\{P_{l}\right\}\right]=\sum_{l}\left(\partial_{\theta} P_{l}\right)^{2} / P_{l}, \hat{\bar{\rho}}_{l}=\hat{\tilde{\rho}}_{l} / P_{l}$ is the normalized reduced density matrix of the system conditioned on the measurement result $l$, and $F_{Q}$ is the single instance QFI given above in Eq. (26). Using this form of the QFI, the Cramer-Rao bound is given by

$$
\overline{\Delta \theta}^{2}=\sum_{l} P_{l}(\Delta \theta)^{2} \geqslant \frac{1}{v \mathbb{F}} .
$$

In our protocol, the conditioned state of the system $\hat{\bar{\rho}}_{l}$ corresponds to the normalized final density matrix of the probe mode $\hat{\rho}_{P}(m)$ exiting from Stage III of the protocol in Fig. 1. We obtain this by scaling the conditioned state by $P(m)$, the probability of our measurement returning a value $m$, i.e., $\hat{\bar{\rho}}(m)=\hat{\tilde{\rho}}(m) / P(m)$, where $\hat{\tilde{\rho}}(m)$ is the final unnormalized postmeasurement probe state at the completion of Stage III in Fig. 1. We can now work out the generalized QFI to be

$$
\mathbb{F}=\int_{-\infty}^{\infty} \frac{\left[\partial_{\theta} P(m)\right]^{2}}{P(m)} d m+\int_{-\infty}^{\infty} P(m) F_{Q}[\hat{\bar{\rho}}(m)] d m,
$$

where the first term is the standard generalized classical FI $\mathbb{F}_{C}$, and the second term is the generalized QFI $\mathbb{F}_{Q}$. To calculate $F_{Q}[\hat{\bar{\rho}}(m)]$, we make use of the fact that, if we consider the normalized postmeasurement probe state, it is in a pure state [see Eq. (14)], allowing us to use Eq. (29). In the analysis below, we find that the classical portion of $\mathbb{F}$ [which only depends on $P(m)$ ] is negligible when compared with the second term, the ensemble averaged QFI, and we will typically focus on the latter. Finally, we mention that the last unitary in Stage III in Fig. 1 is a correction unitary depending on the measurement result. This unitary introduces an additional $\hat{U}_{l}^{C}$ in Eq. (32), and we get $\hat{\rho}_{\text {sys }}(\theta)=\operatorname{Tr}_{E} \hat{\rho}_{\text {ext }}(\theta)=$ $\sum_{l} \hat{U}_{l}^{C} \hat{\Pi}_{l}(\theta) \hat{\rho}_{0} \hat{\Pi}_{l}^{\dagger}(\theta) \hat{U}_{l}^{C \dagger}=\sum_{l} \hat{\tilde{\rho}}_{l}(\theta)$. This does not alter $P(m)$, and except for the change $\hat{\bar{\rho}}_{l} \rightarrow \hat{\bar{\rho}}_{l}^{C} \equiv \hat{U}_{l}^{C} \hat{\bar{\rho}}_{l} \hat{U}_{l}^{C \dagger}$, the above derivation of the generalized QFI proceeds unchanged. We can thus evaluate the generalized QFI of the corrected ensemble using Eq. (35), using $\hat{\bar{\rho}}_{l}^{C}$. In what follows, we drop the $\hat{\tilde{\rho}}$ and $\hat{\bar{\rho}}$ notations for postmeasurement unnormalized or normalized states, referring instead to state vectors $|\Psi\rangle$, which possess nonunit or unit norms.

\section{Super-Heisenberg metrology}

In Sec. II, we observed that the conditioned postmeasurement quantum state $\left|\psi_{I I I}^{M}\right\rangle$ is like Kerr-type evolution of the initial probe state, i.e., $|\psi\rangle_{\text {kerr }} \sim \exp \left(i \chi \hat{n}^{2}\right)|\alpha\rangle$. It is well known that quantum estimation of the strength of the deterministic Kerr can be performed with so-called superHeisenberg scaling in the precision, i.e., $\Delta \chi \sim 1 / \bar{n}^{3 / 2}$ [6], and we now explore how this can be used to perform superHeisenberg metrology of the rotation angle $\theta$, appearing in Stage II of the protocol shown in Fig. 1. We wish to estimate the value of an unknown small deviation $\delta \theta$ of this angle from a preknown bias value, e.g., $\theta=\theta_{0}+\delta \theta$. The protocol involves a position measurement which returns a classical result $m$, with an associated probability distribution $P(m)$, and we will be interested in exploring how the generalized QFI in Eq. (35) scales with the resource $\bar{n}$, the expected Fock number of the input probe state. We recall from Sec. III A that 
shot-noise scaling of $\theta$ corresponds to $\mathbb{F} \sim \bar{n}$, Heisenberg scaling corresponds to $\mathbb{F} \sim \bar{n}^{2}$, while super-Heisenberg corresponds to $\mathbb{F} \sim \bar{n}^{v}$ with $v>2$. Below, we will find that the use of the correction unitary $U_{c}$, shown at the end of Stage III in Fig. 1, plays a crucial role in achieving super-Heisenberg scaling. Without this correction, our protocol achieves a precision worse than shot noise.

To begin, we note that the normalized state $\left|\Psi_{I I I}^{M}\right\rangle$ of the probe after measurement is pure, which enables us to calculate the FI using Eq. (29). Before doing that, we first study the form of the postmeasurement state.

Taking the initial probe state to be $\left|\Psi_{0}\right\rangle_{P}$, which from Eq. (13) and using the homomorphic transformation between the $(\beta, \sigma) \leftrightarrow(\theta, r)$ parametrizations yields

$$
\begin{aligned}
\left|\Psi_{I I I}^{M}\right\rangle & =N_{I I I M}^{\prime} \exp \left[-\frac{\sigma^{2}(g \hat{n}-m)^{2}}{2\left(1+2 i \beta \sigma^{2}\right)}\right]\left|\Psi_{0}\right\rangle_{P} \\
& =N_{I I I M}^{\prime} \exp \left[-f(m, \theta)\left(1-\frac{g}{m} \hat{n}\right)^{2}\right]\left|\Psi_{0}\right\rangle_{P},
\end{aligned}
$$

where

$$
f(m, \theta)=\frac{m^{2}}{4} \frac{1-i e^{2 r} \cot (\theta)}{e^{2 r}-i \cot (\theta)} .
$$

If we now consider the large squeezing limit $r \gg 1$, we find $f(m, \theta) \sim-i m^{2} \cot (\theta) / 4$, and we curiously discover that the effect on the probe mode by the circuit is completely unitary,

$$
\left|\Psi_{I I I}^{M}\right\rangle=\exp \left[\frac{i}{4} \cot (\theta)(m-g \hat{n})^{2}\right]\left|\Psi_{0}\right\rangle_{P},
$$

and thus, in this large squeezing limit, the decoherence channel presented to the probe mode postmeasurement is unital, mapping the identity to itself. In this limit, we observe that the random measurement result $m$ causes a random phase rotation, which more generally depends on $(r, \theta, g, m)$. It is this random phase factor that will prevent us from obtaining optimal scaling of the generalized QFI, as essentially, this is information we are throwing away after each measurement. In the large squeezing limit, if we can apply a unitary correction operation $U_{c}=\exp [\operatorname{igm} \cot (\theta) \hat{n} / 2]$ to the probe state to cancel this phase term, the unknown random measurement effect will be removed. There is an additional global phase which is proportional to $\mathrm{m}^{2}$, but this global phase cannot influence the generalized QFI, as it is not a physical observable in experiments on the postmeasurement state. If we assume we can apply the unitary correction $U_{c}$, the normalized postcorrected pure state in the general case of finite squeezing can be written as

$$
\left|\Psi_{I I I}^{C}\right\rangle=N(m, r, \theta) \exp [f(\theta, \hat{n})]\left|\Psi_{0}\right\rangle_{P},
$$

where

$$
\begin{aligned}
f(\theta, \hat{n})= & \frac{i}{2} g m \hat{n} \cot (\theta) \\
& -\frac{m^{2}}{4}\left[\frac{1-i e^{2 r} \cot (\theta)}{e^{2 r}-i \cot (\theta)}\right]\left(1-\frac{g}{m} \hat{n}\right)^{2},
\end{aligned}
$$

and where the normalization $N(m, r, \theta)$ is taken to be real.

To apply this phase compensation, we note that, as part of our protocol, we already have assumed that we have access to an oracle in Stage II that applies a number-dependent phase shift $R(\varphi) \equiv \exp [i \varphi \hat{n}]$ to the ancilla mode. As mentioned above, we are primarily interested in the estimation of a small unknown $\delta \theta$ about a bias value $\theta_{0}$, i.e., $\varphi=\theta_{0}+\delta \theta$. Since

$$
\cot \left(\theta_{0}+\delta \theta\right)=\cot \theta_{0}-\left(1+\cot ^{2} \theta_{0}\right) \delta \theta+\cdots,
$$

by reusing this oracle, but now operating on the probe mode, we observe that, although we cannot generate the exact $\cot (\theta)$ unitary correction, the oracle, when used with known values of $\varphi$ and the unknown value $\theta=\theta_{0}+\delta \theta$, is capable of applying a compensation to the first order in $\delta \theta$. In this case, the exponent function $f$ is given by

$$
\begin{aligned}
f(\delta \theta, \hat{n})= & \frac{i}{2} g m \hat{n}\left[\cot \theta_{0}-\left(1+\cot ^{2} \theta_{0}\right) \delta \theta\right] \\
& -\frac{m^{2}}{4} \frac{1-i e^{2 r} \cot \left(\theta_{0}+\delta \theta\right)}{e^{2 r}-i \cot \left(\theta_{0}+\delta \theta\right)}\left(1-\frac{g}{m} \hat{n}\right)^{2},
\end{aligned}
$$

where the unknown small parameter to be estimated is now $\delta \theta$. To compute the generalized QFI $\mathbb{F}$, we need to evaluate

$$
\begin{aligned}
\left\langle d \Psi_{I I I}^{C} \mid d \Psi_{I I I}^{C}\right\rangle= & \left|\frac{d N}{N}\right|^{2}+\left(\frac{d N}{N}\right)^{*}\langle d f\rangle+\left(\frac{d N}{N}\right)\langle d f\rangle^{*} \\
& +\left\langle d f^{\dagger} d f\right\rangle \\
\left|\left\langle\Psi_{I I I}^{C} \mid d \Psi_{I I I}^{C}\right\rangle\right|^{2}= & \left|\frac{d N}{N}\right|^{2}+\left(\frac{d N}{N}\right)^{*}\langle d f\rangle+\left(\frac{d N}{N}\right)\langle d f\rangle^{*} \\
& +|\langle d f\rangle|^{2}
\end{aligned}
$$

where $d \equiv d / d(\delta \theta)$, and $f$ is given as in Eq. (42). From this, the pure state QFI is given by

$$
\begin{aligned}
F_{Q}\left(\left|\Psi_{I I I}^{C}\right\rangle\right) & =4\left(\left\langle d \Psi_{I I I}^{C} \mid d \Psi_{I I I}^{C}\right\rangle-\left|\left\langle\Psi_{I I I}^{C} \mid d \Psi_{I I I}^{C}\right\rangle\right|^{2}\right) \\
& =4\left(\left\langle d f^{\dagger} d f\right\rangle-|\langle d f\rangle|^{2}\right) \\
& =F_{Q}\left[\left|\Psi_{I I I}^{C}\right\rangle, f\right]
\end{aligned}
$$

where the expectation values are taken with respect to the normalized state $\left|\Psi_{I I I}^{C}\right\rangle$, with $\delta \theta=0$. This expression can be calculated analytically using Eq. (42), but while straightforward, the resulting expression is lengthy, and for that reason, we do not explicitly include it here. This pure state $F_{Q}$ is a function of $\left(r, \theta_{0}, g, m\right)$, and to obtain the right-hand term of the generalized QFI in Eq. (35), the generalized QFI, we must find a weighted average of $F_{Q}$ over all possible measurement outcomes $m$, yielding

$$
\mathbb{F}_{Q}\left(r, \theta_{0}, g\right)=\int_{-\infty}^{\infty} P(m) F_{Q}\left(r, \theta_{0}, g, m\right) d m,
$$

where $P(m) \equiv\left\langle\Psi_{I I I}^{M} \mid \Psi_{I I I}^{M}\right\rangle$. Assuming, as we did in Sec. II, that the input probe state is a coherent state $\left|\Psi_{0}\right\rangle_{P}=|\alpha\rangle$, with expected Fock number $\bar{n}_{P}$, we find that, although the expressions in Eq. (48) and $P(m)$ can be found analytically in terms of infinite summations, they cannot be evaluated analytically except in the simplest cases, and we thus evaluate them numerically. We note that this can be difficult, as one must check convergence of these numerical expressions both with the Fock number truncation and also with the precision in numerical accuracy, as the integrand in Eq. (48) can oscillate rapidly in cases and is often vanishing outside a compact domain in $m$. 

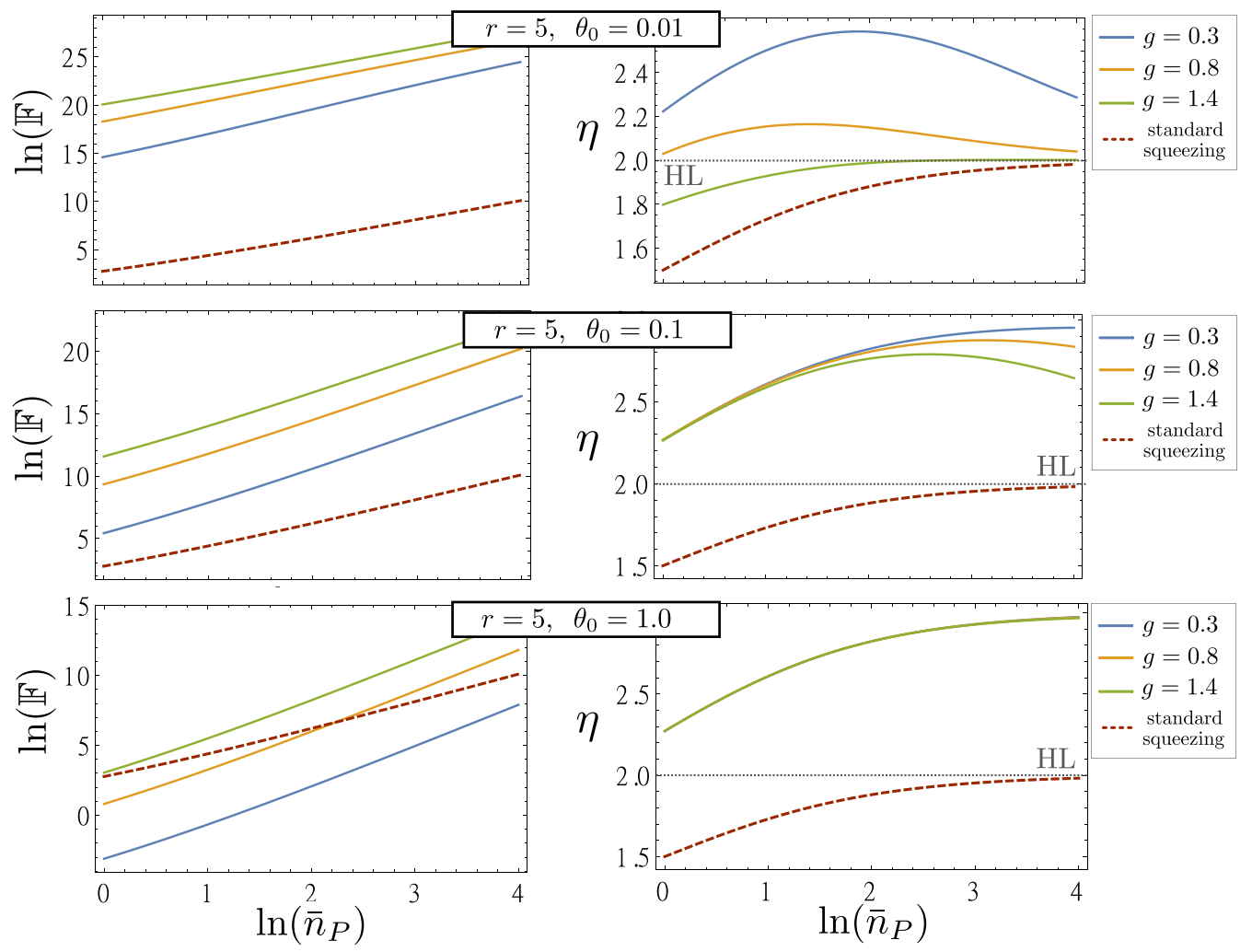

FIG. 2. Plot of the generalized Fisher information (FI) for phase estimation $\mathbb{F}$ (left) and the exponent scaling $\eta$ of $\mathbb{F} \sim \bar{n}_{P}^{\eta}$ (right) as a function of the probe mode mean Fock number $\bar{n}_{P}$ of our scheme and for standard metrology using a squeezed input probe state with an identical $\bar{n}_{P}$. We consider various cross-rotation coupling strengths $g$, ancilla mode squeezing strengths $r$, and bias phase shifts $\theta_{0}$. The curves shown in the right panels are the slopes of the curves shown in the left panels. We take the horizontal axis to be the natural logarithm of $\bar{n}_{P}$, the mean Fock number of the input probe coherent state, and in the case of standard squeezing, it is the mean Fock number of the input squeezed state. The curves colored blue, orange, green correspond to $g=(0.3,0.8,1.4)$, respectively, while the red curves correspond to standard squeezing. Note that, in the bottom right plot, the blue, orange, and green curves lie on top of each other. Super-Heisenberg scaling corresponds to $\eta>2$, and from the right panels, we observe many situations which achieve this for our protocol. In these numerics, we set the Fock truncation to be $N_{\text {trunc }}=260$ and truncate the numerical integration along the $m$-axis to be within the domain $m \in$ [-900,900].

We are now in a position to examine the scaling of the generalized FI $F$ in Eq. (35) to estimate $\delta \theta$ using our protocol. We assume a coherent probe state input $\left|\Psi_{0}\right\rangle_{P}=|\alpha\rangle$, with expected Fock number $\bar{n}_{P}$, and assume that, while we cannot carry out a full $\cot (\theta)$ phase compensation as in Eq. (40), we can cancel the phase to first order, as in Eq. (42).

We begin by examining how the QFI $\mathbb{F}\left(r, \theta_{0}, g\right)$ scales with respect to the average Fock number $\bar{n}_{P}$ of the probe. Figure 2 shows the behavior of $\mathbb{F}$ for various values of $\theta_{0}$, coupling strength $g$, and squeezing $r$. We also graph the exponent $\eta$, given by $\mathbb{F} \sim \bar{n}_{P}^{\eta}$, by defining $\eta=d(\ln \mathbb{F}) / d\left(\ln \bar{n}_{P}\right)$. Recall that the SQL, Heisenberg, and super-Heisenberg scalings correspond to $\eta=1.0,2.0,>2.0$, respectively. During the numerical evaluations, we discover that $\mathbb{F}_{C} \ll \mathbb{F}_{Q}$, and so $\mathbb{F} \sim \mathbb{F}_{Q}$ from Eq. (35). From Fig. 2, two things are clear: First, $\mathbb{F}$ is much higher than if we performed the estimation of $\delta \theta$ using the ancilla mode alone when prepared in a standard squeezed state with the same value of $\bar{n}_{P}$. Second, the actual scaling with $\bar{n}_{P}$ is better than that found using a squeezed state resource, and so the scaling in the FI is super-Heisenberg. Using a squeezed state resource asymptotically approaches $\mathbb{F} \sim \bar{n}_{P}^{2}$ for large $\bar{n}_{P}$, whereas our scheme approaches $\mathbb{F} \sim \bar{n}_{P}^{3}$.
We also note the role of the bias angle $\theta_{0}$, as one may wish to choose values of the angle $\theta_{0}$ which maximize the FI. Results for $\theta_{0}=(0.01,0.1,1.0)$ rad, are shown in Fig. 2. From this, we observe that, as $\theta_{0} \rightarrow 0$, we obtain a much higher absolute value of the generalized FI $\mathbb{F}$, but the scaling of $\mathbb{F}$ with $\bar{n}_{P}$ weakens and does not hold over the same large range of $\bar{n}_{P}$. We also study how the generalized QFI depends on the ancilla mode squeezing $r$. This is shown in Fig. 3. As the squeezing $r$ is increased, we obtain both higher absolute FI as well as better scaling, although the gains do saturate. One obvious question is how well the linear correction scheme works; that is, how well does the generalized QFI resulting from Eq. (42) compare with the case when we just throw that information away and do not apply the correction unitary $U_{c}$ at all, i.e., taking the final state of the protocol to be Eq. (36)? This is plotted in Fig. 4. As before, a smaller $\theta_{0}$ results in a higher absolute $\mathbb{F}$ but worse scaling. We also see that, without any correction, in no case does the protocol scale better than the shot noise limit. However, with correction, we achieve superHeisenberg scaling and reach a total FI greater than what can be obtained from using a standard squeezed state with the same $\bar{n}_{P}$. 

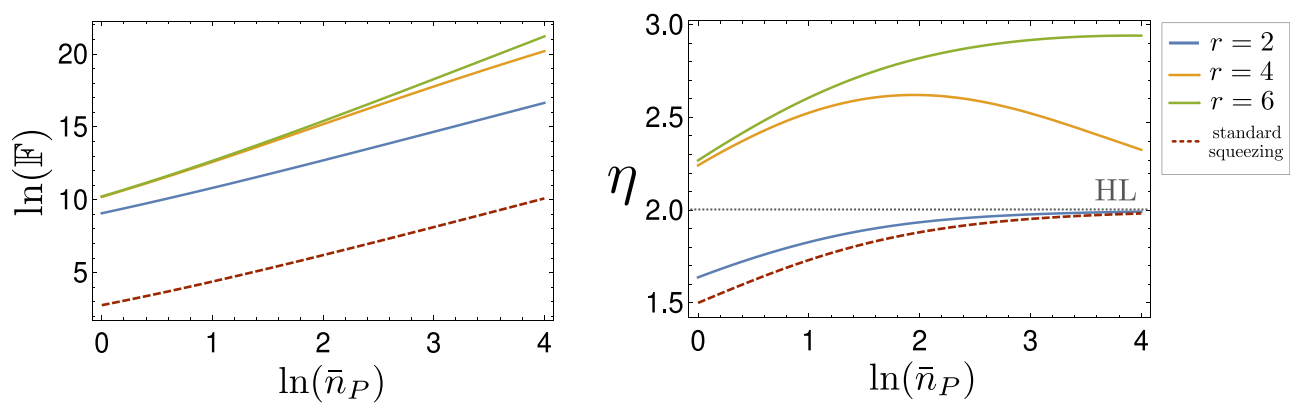

FIG. 3. Plot of the generalized quantum Fisher information (QFI) for the linearly corrected scheme for various values of ancilla mode squeezing $r$ compared with the case of Heisenberg scaling using a standard squeezed state with the same $\bar{n}_{P}$. Curves colored blue, orange, green correspond to $r=(2,4,6)$, respectively, with $\theta_{0}=0.1, g=1.0$. The red curve corresponds to standard squeezing.

In summary, we have numerically evaluated the generalized FI for the linear corrected metrology protocol and find that, for a range of coupling strengths $g$ and bias angles $\theta_{0}$, the protocol exhibits $\mathbb{F} \sim \bar{n}_{P}^{\eta}$, where $\eta>2$, or super-Heisenberg scaling. The central idea behind how this is achieved is essentially via the integral in Eq. (12), which effectively squares $b$ in the exponential. By applying the cross-rotation operator $\exp \left(-i g \hat{p}_{A} \otimes \hat{n}_{P}\right)$ in Stage III, we end up with a Kerr-type evolution on the probe mode $\sim \exp \left[i g^{2} / 4 \cot (\theta) \hat{n}^{2}\right]$ in the limit of large ancilla mode squeezing. Following this logic, if instead we applied the cross-mode operation $\exp \left(-i g \hat{p} \otimes \hat{A}_{P}\right)$, then we would end up applying the operation $\exp \left[i g^{2} / 4 \cot (\theta) \hat{A}^{2}\right]$ on the probe mode for $r \gg 1$. We now briefly describe how this observation can be used to adapt the circuit shown in Fig. 1 to bootstrap up other metrology protocols to improve the scaling of their FI, effectively surpassing their normal Heisenberg metrology limits.

\section{Bootstrap protocol}

As mentioned above, the protocol described in Fig. 1 can essentially generate a Kraus operator on a target mode which is generated by the square of the target operator in the two-
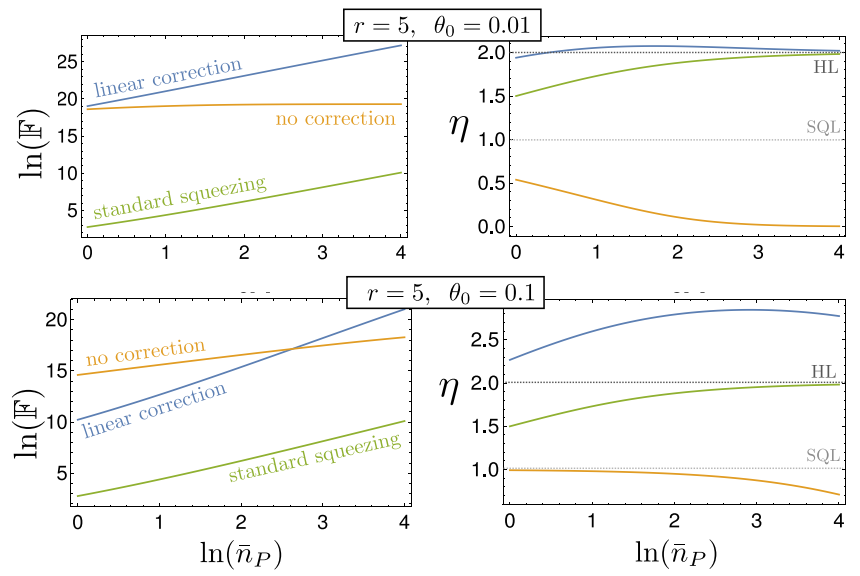

FIG. 4. Comparison of how well the linear correction scheme does when compared to the non-corrected scheme. Shown is the generalised Fisher information $\mathbb{F}$ (left column) and the scaling exponent $\eta$ (right column). The (orange, green, blue) curves indicate (no correction, standard squeezing, linear correction), with $g=1.0$. mode gate $C_{R}$. In quantum metrology, one effectively wishes to estimate a parameter $\theta$, which naturally appears in some unitary $U(\theta)=\exp (i \theta \hat{G})$, via the action of this unitary on a state $\left|\psi_{\theta}\right\rangle=U(\theta)\left|\psi_{0}\right\rangle$. The scaling of the QFI effectively is given by the uncertainty of $\hat{G}$ with respect to $\left|\psi_{0}\right\rangle$ [see Eq. (30)]. The protocol in Fig. 1 effectively can "boot up" the power of $\hat{G}$ appearing in the unitary $U$, e.g., $\exp (i \theta \hat{G}) \rightarrow$ $\exp \left[i h(\theta) \hat{G}^{2}\right]$, where the function $h$ may be determined. The scaling of the QFI to estimate $\theta$ after this boot up will now be determined by the uncertainty of $\hat{G}^{2}$, which will scale with a larger power of the resource than $\hat{G}$. In the above, we have used the probe occupation number as the basis for our resource counting, as the probe mode is often the experimentally adjustable component, while the elements in the ancilla are held fixed. Using this insight, we now show how to adapt the protocol described in Sec. III C to estimate the parametergenerating displacements in phase space with an imprecision which scales better than the standard HL. Such displacement estimation is a crucial ingredient for many force sensing schemes. In this case, $\left|\psi_{\kappa}\right\rangle \equiv \exp (i \kappa \hat{p})\left|\psi_{0}\right\rangle$, where the base state $\left|\psi_{0}\right\rangle$ has mean Fock number $\bar{n}$. The HL for displacement measurements is achieved when $\left|\psi_{0}\right\rangle$ is a squeezed state or compass state [34], and in that case, the QFI achieves a scaling of the form $F_{Q}(\kappa) \sim \bar{n}$. By using the bootstrapped scheme we outline below, we argue we can achieve $F_{Q}(\kappa) \sim \bar{n}^{3}$ for the QFI in displacement sensing. This would yield an imprecision which scales as $\Delta \kappa \sim 1 / \sqrt{\bar{n}^{3}}$, rather than $\Delta \kappa \sim 1 / \sqrt{\bar{n}}$, providing a vast improvement of the accuracy for displacement or force sensing.

We focus on estimating the parameter $\kappa$ in the single-mode displacement operator $D(\kappa)_{A 1}=\exp \left(i \kappa \hat{p}_{A 1}\right)$. This operator displaces the mode $A 1$ along the $\hat{q}_{A 1}$ quadrature. We now assume we can access the two-mode gate $\exp \left(i \kappa \hat{p}_{A 1} \otimes \hat{p}_{A 2}\right)$. Having access to this gate, we can consider the bootstrap circuit depicted in Fig. 5. The lower section of this circuit is Stages III and IV of the original circuit of Fig. 1. The top circuit in Fig. 5 is a replication of the original protocol but with some changes. Following the derivations in Sec. II, we see that, in the large squeezing limit for mode $A 1$, i.e., when $r^{\prime} \gg 1$, and when $\theta^{\prime}=\pi / 4$, we have

$$
\left|\Psi_{\text {out }}\right\rangle_{A 2}=\exp \left(\frac{i}{4} \kappa^{2} \hat{p}_{2}^{2}\right) S(r)|0\rangle_{A 2} .
$$

We now return to Eq. (11) and note that, when $\left|\Psi_{\text {out }}\right\rangle_{A 2}$ is input into Stages III and IV of the original circuit, it remains 


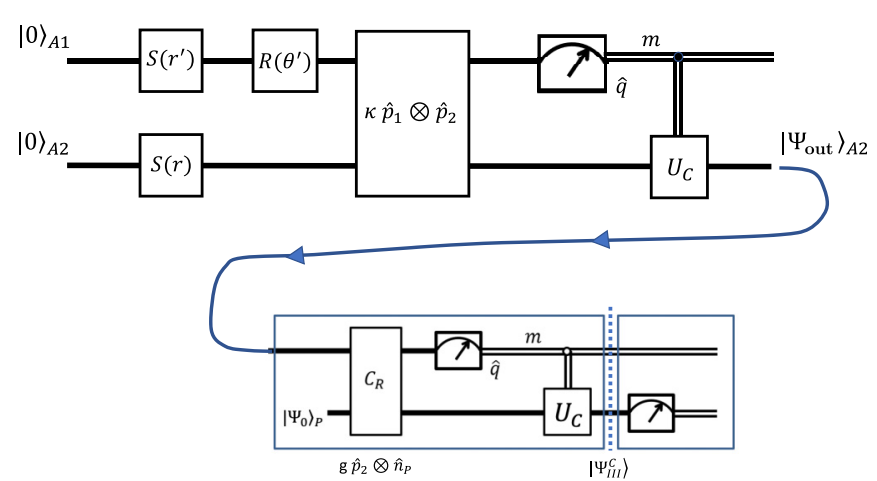

FIG. 5. Schematic of the bootstrap scheme to estimate the parameter $\kappa$, generating displacements in phase space.

unchanged except for $\beta \rightarrow-\kappa$. As the original protocol operating with linear correction can provide a generalized QFI for $\theta$ (or $\beta$ ) which scales as $\bar{n}^{\nu}$, with $v \in(2-3$ ), we expect to find a similar scaling for the estimate of $\kappa$. In the example, as depicted in Fig. 5, we described how to teleport the displacement parameter $\kappa$ into the strength of the Kerr dynamics, whose metrology can achieve super-Heisenberg scaling with respect to the probe occupation when we can correct for the random unitary rotations caused by the probabilistic measurement. If we replace the upper quantum circuit of Fig. 5 with Stages I, II, and III from Fig. 1, we could aim to effectively generate an action on the probe which is the square of the Kerr, i.e., $\sim\left(\hat{a}^{\dagger} \hat{a}\right)^{2}$, and if we could generate this, we may achieve metrology which scales as $\delta \theta \sim \bar{n}^{5 / 2}$. However, to achieve this type of scaling would require the correction unitary $U_{c}$ to correct for random Kerr rotations on the probe, which would involve further iterations of circuits like Fig. 1, bringing further loss in fidelity and greatly increased complexity to the resulting overall quantum circuit. Thus, although in principle this booting up beyond Kerr could be possible, it seems highly infeasible in practice.

\section{APPLICATION TO MAKE CAT STATES AND COMPASS STATES}

We now return to the basic protocol outlined in Fig. 1 but now assume we have full knowledge of all the parameters in the circuit, particularly the rotation angle $\theta$ in Stage II. In this situation, we study how well this circuit can be used to generate highly nonclassical quantum states of the probe mode. Cat states and compass states [34] have been proposed for quantum computation and for precision sensing of displacement [47]. Such states exhibit fine detail in their Wigner functions, and small displacements of such states become rapidly orthogonal. Here, we show how our protocol allows deterministic preparation of high-fidelity cat and compass states.

To see this, we go back to the output state of the general protocol in Eqs. (14)-(18),

$$
\left|\Psi_{\text {out }}\right\rangle=\mathcal{N} U(\beta) U_{c}(\beta, m) K(\beta) K_{c}(\beta, m)\left|\Psi_{0}\right\rangle,
$$

where, again, we have decomposed the action of the channel into four distinct operations. Recall that the form of the conditioned unitary $U_{c}$ is

$$
U_{c}=\exp \left(i \frac{m g}{2} \frac{v}{u^{2}+v^{2}} \hat{n}\right) \equiv \exp \left[i \phi_{c}(m) \hat{n}\right]
$$

where we have made use of the holomorphic parametrization in Eq. (20). As mentioned before, we will assume that $(\theta, r, g)$ are all controllable known parameters, and thus, although the precise values of the measurement result $m$ and the phase $\phi_{c}(m)$ are random in each execution of the protocol, they both are known precisely. As a consequence, the operation $U_{c}$ can be undone exactly independent of the initial state and the degree of squeezing in the ancilla mode. Furthermore, in the high-squeezing limit, where $r \rightarrow \infty$, we have $K, K_{c} \sim \mathbb{I}$, and so the evolution ends up being dictated solely by the unconditioned unitary $U(\theta)$ :

$$
U(\theta)=\exp \left[-i \gamma(\theta) \hat{n}^{2}\right], \quad \text { with } \quad \gamma(\theta)=\frac{g^{2} v}{4\left(u^{2}+v^{2}\right)} .
$$

Taking the initial probe state to be a coherent state, we have that $\left|\Psi_{\text {out }}\right\rangle_{P} \simeq U(\theta)|\alpha\rangle_{P}$, and thus, a judicious choice of $\gamma(\theta)$ will yield states such as

$$
\begin{aligned}
\gamma(\theta) & =\frac{\pi}{2} \Rightarrow \exp \left(-i \frac{\pi}{2} \hat{n}^{2}\right)|\alpha\rangle \\
& \left.=\frac{\exp \left(-i \frac{\pi}{4}\right)}{\sqrt{2}}(|\alpha\rangle+i|-\alpha\rangle) \equiv \mid \text { cat }\right\rangle, \\
\gamma(\theta) & =\frac{\pi}{4} \Rightarrow \exp \left(-i \frac{\pi}{4} \hat{n}^{2}\right)|\alpha\rangle \\
& =\frac{1}{2}\left[\exp \left(i \frac{\pi}{4}\right)(|\alpha\rangle-|-\alpha\rangle)+(|i \alpha\rangle+|-i \alpha\rangle)\right] \\
& \equiv \mid \text { compass }\rangle,
\end{aligned}
$$

where we have dropped the probe $P$ subscript. In the following, we will study how well we can achieve these ideal target states in the case of large but finite squeezing in the ancilla mode.

\section{A. Choice of parameter $\boldsymbol{\theta}$ and minimum squeezing requirements}

For finite squeezing, the nonunitary part of the evolution in Eq. (50) will impact how well we can prepare the desired target states. Before we analyze such an impact, we must determine under which circumstances it is a priori possible to set the parameters of the evolution as in Eqs. (53) and (54). For this, we can refer back to Eqs. (20) and (52) to find

$$
\gamma(\theta)=\frac{g^{2}}{4} \frac{\left(1-e^{4 r}\right) \cot (\theta)}{e^{4 r}+\cot ^{2}(\theta)},
$$

and for $r \gg 1$, we obtain $\gamma=-g^{2} \cot (\theta) / 4$. For finite $r$, we notice that $\gamma(\theta)$ is an odd function about the value $\theta=\pi / 2$ and obeys $\gamma(0)=\gamma(\pi / 2)=0$, and thus, it reaches a maximum absolute value $\gamma_{\max }=\gamma\left(\theta_{c}\right)$ in the interval $\theta \in[0, \pi / 2]$. For fixed coupling strength $g$, the magnitude of $\gamma_{\max }$ increases with the amount of squeezing $r$ in the ancillary mode, as 

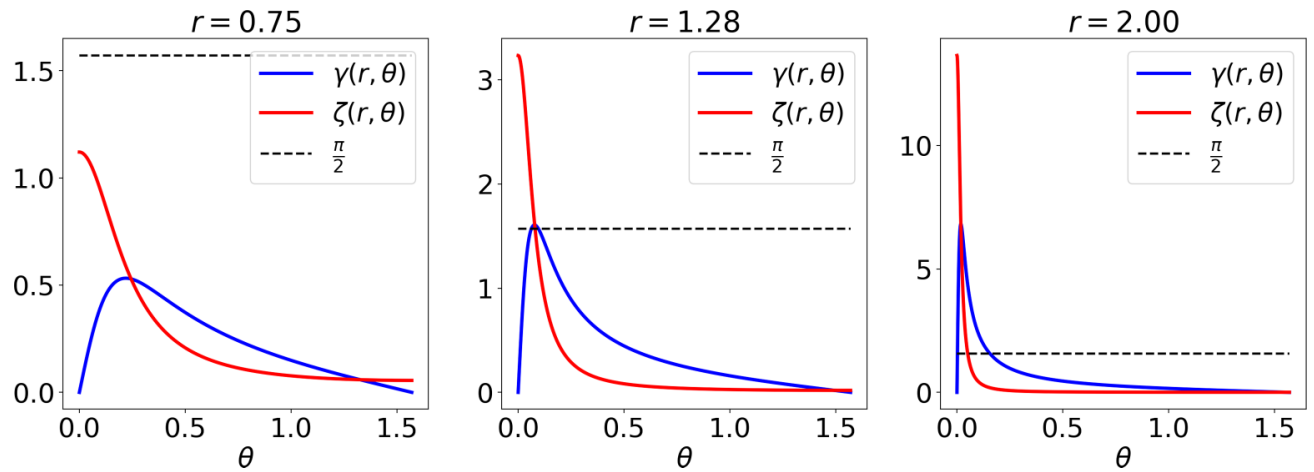

FIG. 6. Plots of functions $\gamma(\theta)$ and $\zeta(\theta)$ defined in Eqs. (52) and (60) as a function of parameter $\theta$ and for different values of the squeezing parameter $r$ (here $g=1)$. The parameter $\zeta(r, \theta)$ describes the nonunitary part of the process. We observe that $\gamma=\pi / 2(\pi / 4)$ can be achieved only if $r \gtrsim 1.27(0.93)$.

depicted in Fig. 6. This means that, to set the protocol parameters as required by Eqs. (53) and (54), a certain minimum squeezing value is required. One can derive an expression for $\gamma_{\max }$, which leads to the following squeezing thresholds (obtained numerically):

$$
\begin{array}{r}
\text { |compass }\rangle: \frac{\gamma_{\max }}{g^{2}} \geqslant \frac{\pi}{4} \Leftrightarrow r>0.93, \\
\mid \text { cat }\rangle: \frac{\gamma_{\max }}{g^{2}} \geqslant \frac{\pi}{2} \Leftrightarrow r>1.27 .
\end{array}
$$

In the remainder of this section, we take $g=1$ without loss of generality and consider values of $r$ equal or greater than these threshold values, which allows us to $\operatorname{set} \theta=\theta_{*}$ such that $\gamma\left(\theta_{*}\right)=\frac{\pi}{4}$ or $\frac{\pi}{2}$, depending on the target state.

\section{B. Effects of nonunitary backaction and conditioned state preparation fidelity}

We now turn to analyzing how well our protocol can prepare to the target states of interest. We first define the (conditioned) state fidelity $\mathcal{F}_{m}$ as

$$
\mathcal{F}_{m}=\left|\left\langle\operatorname{target} \mid \Psi_{\text {out }}\right\rangle\right|^{2},
$$

where $|\operatorname{target}\rangle=\exp \left[-\mathrm{i} \gamma\left(\theta_{*}\right) \hat{\mathrm{n}}^{2}\right]|\alpha\rangle$ refers to the target states in Eqs. (53) and (54) and recall that $\theta_{*}$ is chosen to give $\gamma\left(\theta_{*}\right)=\frac{\pi}{2}$ or $\frac{\pi}{4}$ (depending on the particular target). Using Eq. (50), the fidelity can be cast directly in terms of the operators of interest:

$$
\begin{aligned}
\mathcal{F}_{m} & =|\mathcal{N}|^{2}\left|\left\langle\alpha\left|U\left(\theta_{*}\right)^{\dagger} U\left(\theta_{*}\right) K\left(\theta_{*}\right) K_{c}\left(m, \theta_{*}\right)\right| \alpha\right\rangle\right|^{2} \\
& =\frac{\left|\left\langle\alpha\left|K\left(\theta_{*}\right) K_{c}\left(m, \theta_{*}\right)\right| \alpha\right\rangle\right|^{2}}{\left\langle\alpha\left|K\left(\theta_{*}\right)^{2} K_{c}\left(m, \theta_{*}\right)^{2}\right| \alpha\right\rangle},
\end{aligned}
$$

where we have used $\left\langle\Psi_{\text {out }} \mid \Psi_{\text {out }}\right\rangle=1$. To analyze the effects of finite squeezing, we recast the nonunitary part of Eq. (50) in the following form:

$$
\begin{aligned}
K(\theta) K_{c}(m, \theta) & =\exp \left[-\zeta(\theta) \hat{N}_{m}\right], \\
\text { with } \quad \hat{N}_{m} & \equiv \hat{n}^{2}-\frac{2 m}{g} \hat{n} \quad \text { and } \quad \zeta(\theta)=\frac{g^{2} u}{4\left(u^{2}+v^{2}\right)},
\end{aligned}
$$

where the function $\zeta(\theta)$, complementary to $\gamma(\theta)$, monotonically decays from $\zeta(0)=g^{2} e^{2 r} / 4$ to $\zeta\left(\frac{\pi}{2}\right)=g^{2} e^{-2 r} / 4$ and is shown in Fig. 6 for various values of $r$. For a given level of squeezing, setting $\theta_{*}$, as described above, fixes the value of $\zeta\left(\theta_{*}\right)$, which for large $r$ we expect to behave like $\zeta\left(\theta_{*}\right) \equiv$ $\zeta \sim e^{-2 r} \ll 1$. We can then expand the operator in Eq. (60) in powers of $\zeta, \exp \left(-\zeta \hat{N}_{m}\right) \simeq 1-\zeta \hat{N}_{m}+\zeta^{2} \hat{N}_{m}^{2} / 2$. Replacing this in Eq. (59), we obtain

$$
\mathcal{F}_{m} \simeq \frac{\left(1-\zeta\left\langle\hat{N}_{m}\right\rangle+\frac{\zeta^{2}}{2}\left\langle\hat{N}_{m}^{2}\right\rangle\right)^{2}}{1-2 \zeta\left\langle\hat{N}_{m}\right\rangle+2 \zeta^{2}\left\langle\hat{N}_{m}^{2}\right\rangle},
$$

where the expectation values are taken over the initial coherent state $|\alpha\rangle$. Keeping the leading order contribution, we finally get

$$
\mathcal{F}_{m} \simeq 1-\zeta^{2}\left(\left\langle\hat{N}_{m}^{2}\right\rangle-\left\langle\hat{N}_{m}\right\rangle^{2}\right)=1-\left.\zeta^{2}\left(\Delta \hat{N}_{m}\right)\right|_{\alpha} ^{2} .
$$

\section{Average fidelity and numerical results}

The fidelity in Eq. (62) still depends on the random measurement outcome $m$, which in turn is a function of the parameters of the protocol, including the squeezing $r$ in the ancilla mode. To obtain a clearer picture about the target state preparation fidelity in our protocol, we consider the average (unconditioned) fidelity

$$
\mathcal{F}_{\text {avg }}=\int d m P(m) \mathcal{F}_{m},
$$

where $P(m)$ is the probability distribution associated with the random measurement outcome $m$, i.e.,

$$
\begin{aligned}
P(m) & =\mathcal{A}\left\langle\alpha\left|K_{0}\left(\theta_{*}\right)^{2} K_{c}\left(m, \theta_{*}\right)^{2}\right| \alpha\right\rangle \\
& =\sqrt{\frac{2 \zeta}{\pi g^{2}}} e^{-|\alpha|^{2}} \sum_{n} \frac{|\alpha|^{2 n}}{n !} \exp \left[-2 \zeta\left(n-\frac{m}{g}\right)^{2}\right] .
\end{aligned}
$$

Since the conditioned fidelity is quadratic in $m$, to compute the average fidelity, we need to compute the first two moments of this distribution $\bar{m}$ and $\overline{m^{2}}$. These can be computed easily thanks to the Gaussian form of each term in Eq. (64). For the 

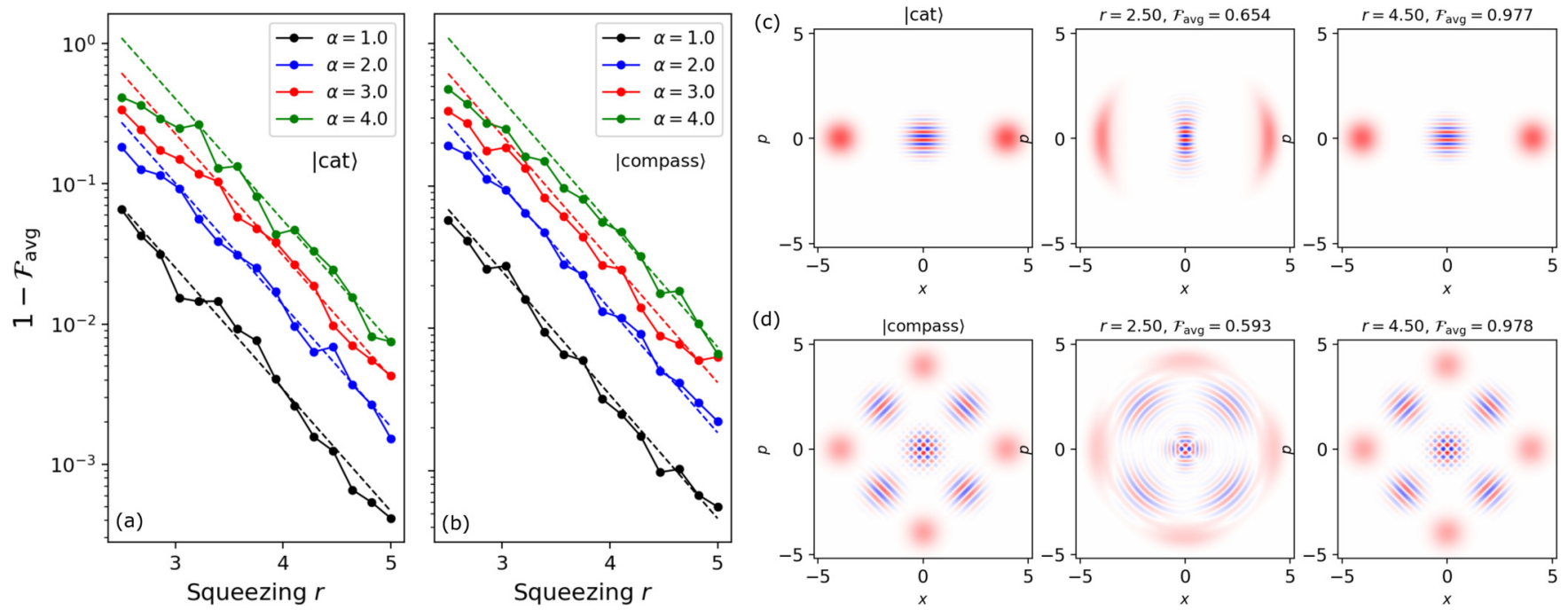

FIG. 7. Cat and compass state generation with the proposed protocol. (a) and (b) The average state infidelity $1-\mathcal{F}_{\text {avg }}$ computed numerically as a function of the squeezing magnitude $r$ of the ancillary mode. Each value is obtained by averaging over 50 runs of the protocol. Results are shown for different values of initial coherent state amplitude $\alpha$ and for the cases where the target state is (a) |cat $\rangle$ and (b) $\mid$ compass $\rangle$. Dashed lines indicate the leading-order analytical estimate of Eq. (68). (c) and (d) Wigner function of the ideal target states (left plots) and of the states generated by the protocol when $r=2.5$ (center) and $r=4.5$ (right). Results for Wigner functions are obtained from averages over 10 realizations of the protocol in each case, and the value of the coherent state amplitude is set to $\alpha=4$.

mean value, we have

$$
\begin{aligned}
\bar{m}= & \int m P(m) d m=\sqrt{\frac{2 \zeta}{\pi g^{2}}} e^{-|\alpha|^{2}} \sum_{n} \frac{|\alpha|^{2 n}}{n !} \\
& \times \int m \exp \left[-2 \zeta\left(n-\frac{m}{g}\right)^{2}\right] d m \\
= & g e^{-|\alpha|^{2}} \sum_{n} n \frac{|\alpha|^{2 n}}{n !}=g e^{-|\alpha|^{2}} \sum_{m} \frac{|\alpha|^{2 m}}{m !}|\alpha|^{2}=g|\alpha|^{2} .
\end{aligned}
$$

Using similar techniques, we can calculate the second moment, for which we obtain

$$
\overline{m^{2}}=g^{2}\left[\frac{1}{4 \zeta}+|\alpha|^{2}\left(1+|\alpha|^{2}\right)\right] .
$$

With these results, we can combine Eq. (59) with Eq. (63) to obtain

$$
\mathcal{F}_{\text {avg }}=1-\zeta|\alpha|^{2}+\mathcal{O}\left(\zeta^{2}\right)
$$

Notice that, due to the $\zeta^{-1}$ dependence of $\overline{m^{2}}$, the leadingorder contribution of the average fidelity is $\mathcal{O}(\zeta)$. The asymptotic expression in Eq. (68), valid for large squeezing $r$, is the main result of this section. It shows that, in this regime, we expect the fidelity of cat and compass state preparation to increase exponentially with the ancilla squeezing $r$ since $\zeta \sim e^{-2 r}$. For fixed squeezing, however, the average fidelity drops linearly with the mean Fock number of the original probe coherent state $\bar{n}=|\alpha|^{2}$.

To test these results, we performed numerical simulations of the cat and compass state preparation protocol. Results are shown in Fig. 7. In (a) and (b), we show the average infidelity $1-\mathcal{F}_{\text {avg }}$ computed over 50 runs of the protocol for different values of initial coherent state amplitude. The results clearly show that the infidelity drops exponentially with the squeezing magnitude $r$, in excellent agreement with the analytical result of Eq. (68), even for moderate values of $r$. We observe that, for small $r$ (large $\zeta$ ), fidelities are higher than those predicted by the leading-order calculation, indicating that the protocol behaves better than expected in this regime. The actual states achieved by the protocol are depicted in Figs. 7(c) and 7(d), where we plot the Wigner functions of the resulting states for the ideal $(r \rightarrow \infty)$ and the finite squeezing cases.

\section{PHYSICAL IMPLEMENTATION OF OUR SCHEME}

In the above, we have described how to effectively generate nonlinear dynamics via the protocol described in Fig. 1. We showed how it can be used to perform precision measurement and to synthesise nonclassical quantum states. One of the central ingredients to our scheme is the two mode cross-rotation gate shown in Fig. 1. Such conditional dynamics may be possible in optomechanical systems where the single-photon interaction is $\hat{H}_{\mathrm{int}}=-g \hat{x} \otimes \hat{a}^{\dagger} \hat{a}$, but this would require large single-photon optomechanical coupling strengths. A continuous variable universal gate set in superconducting microwave quantum optics has recently been developed [48], and this should be capable of implementing our protocol. However, we focus here on outlining a potential physical implementation of our protocol using atomic ensembles interacting with light through the Faraday effect. We describe the single atomic ensemble as a bosonic mode via the Holstein-Primakoff approximation [49], making the assumption that the ensemble is made up of very many $(N \gg 1)$ atomic spins and that the state stored in the ensemble has a close to maximal $R^{\dagger} J_{Z} R$ expectation value for some $R \in \mathrm{SU}(2)$ and where $J_{K}=\sum_{\alpha=1}^{N} \sigma_{K}^{(\alpha)}$ is the component of the collective angular momentum in the direction $K(K=X, Y, Z)$. Spin basis states are mapped to 


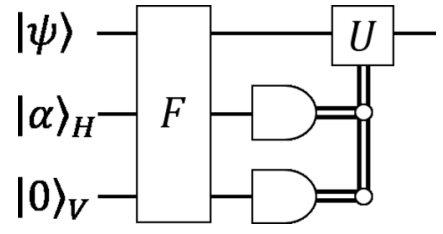

FIG. 8. Gadget for measuring the spin- $Z$ direction of the ensemble in state $|\psi\rangle$. This is coupled to a pair of light modes (one for each polarization) by the Faraday interaction $H_{F}=\chi J_{Z} S_{Y}$. Then we use photodetectors to measure how much the polarization of the light has shifted, thereby approximating the $Y$ component of the angular momentum of $|\phi\rangle$.

bosonic Fock states via

$$
R^{\dagger}|j, j-m\rangle_{Z} \mapsto \frac{1}{\sqrt{m !}}\left(\hat{a}^{\dagger}\right)^{m}|0\rangle,
$$

and spin observables to bosonic observables via

$$
\begin{aligned}
& R^{\dagger} J_{+} R=\sqrt{2 j} \sqrt{1-\frac{\hat{a}^{\dagger} \hat{a}}{2 j}} \hat{a} \approx \sqrt{2 j} \hat{a}, \\
& R^{\dagger} J_{-} R=\sqrt{2 j} \hat{a}^{\dagger} \sqrt{1-\frac{\hat{a}^{\dagger} \hat{a}}{2 j}} \approx \sqrt{2 j} \hat{a}^{\dagger}, \\
& R^{\dagger} J_{Z} R=j-\hat{a}^{\dagger} \hat{a},
\end{aligned}
$$

where the approximation sign holds for large $j=N / 2$. Our protocol requires two fundamental operations. The first involves implementing an $\mathrm{SU}(2)$ rotation using a magnetic field via a Zeeman interaction [50], i.e., $\vec{B} \cdot \vec{J}$. The second uses the Faraday interaction $H_{F}=\chi J_{Z} S_{Y}$ to couple the $J_{Z}$ component of the angular momentum of the ensemble to polarization degree of freedom of light, which is described in terms of the Stokes vector operators: $S_{j}=\frac{1}{2} \mathbf{a}^{\mathrm{H}} \sigma_{j} \mathbf{a}$ [here, we use $\mathbf{a}=$ $\left(\hat{a}_{H}, \hat{a}_{V}\right)^{\mathrm{T}}$ and $\left.\mathbf{a}^{\mathrm{H}}=\left(\hat{a}_{H}^{\dagger}, \hat{a}_{V}^{\dagger}\right)\right]$.

We can prepare the ensemble in a momentumsqueezed state [in the Holstein-Primakoff approximation centered at the positive $X$ axis, achieved by setting $\left.R=\exp \left(-i \pi J_{Y} / 4\right) \exp \left(-i \pi J_{X} / 4\right)\right]$, by implementing $H_{F}$ and subsequently measuring the change in the plane of polarization of the light, as shown in the circuit of Fig. 8. This produces a sequence of weak measurements of $J_{Z}$, which are represented by the following Kraus operator applied to the state [51,52]:

$$
K_{m}=\frac{1}{\left(2 \pi \sigma^{2}\right)^{1 / 4}} \exp \left[-\frac{1}{4 \sigma^{2}}\left(J_{Z}-m\right)^{2}\right],
$$

where $\sigma$ is the measurement resolution, which is related to the interaction strength $\chi$ and the measurement time $\Delta t$. In the Holstein-Primakoff picture, this will correspond to a displaced momentum-squeezed state. We transform this into a position-squeezed vacuum state by correcting the measurement-dependent displacement using $\exp \left(\right.$ it $\left.\frac{J_{Y}}{2 \sqrt{j}}\right) \mapsto$ $\exp (-i t \hat{p})$, followed by a $90^{\circ}$ rotation about the $X$ axis, which implements a Fourier transform in the Holstein-Primakoff mode picture. We can squeeze the input substantially if

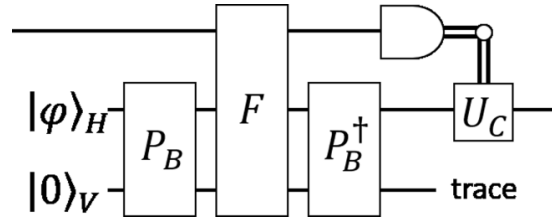

FIG. 9. We teleport the information from the ensemble onto the optical state in the $H$ mode. We can put this mode in any input state $|\varphi\rangle$ (which might be in a coherent state), and we do a $C_{R}$ operation by sandwiching the Faraday interaction between a pair of beam splitters and rotations. Finally, we measure the ensemble along the $Z$ direction, which gives us information about the $\hat{q}$ quadrature in the Holstein-Primakoff approximation. Note that this measurement may itself require coupling to additional light modes. We also allow for a postmeasurement unitary conditioned on the outcomes.

$e^{-2 r} \approx \sigma^{2} / j \ll 1$. After the preparation of a squeezed state, the ensemble interacts with the signal present in the magnetic field via the Zeeman interaction:

$$
B J_{X}=B R^{\dagger} J_{Z} R \mapsto B\left(j-\hat{a}^{\dagger} \hat{a}\right) .
$$

In the Holstein-Primakoff picture of the ensemble as a bosonic mode, this acts as a single-mode phase gate with $\theta=B$. We next use the Faraday interaction $H_{F}=\chi J_{Z} S_{Y}$ to implement the $C_{R}$ gate (which is given in Fig. 1, by $C_{R}=\exp \left(i g \hat{p}_{A} \otimes \hat{n}_{P}\right)$. We do this as shown in Fig. 9.

We use a polarizing beam splitter $P_{B}=\exp \left[i \pi\left(\hat{a}_{H}^{\dagger} a_{V}+\right.\right.$ $\left.\left.\hat{a}_{V}^{\dagger} a_{H}\right) / 2\right]$ to rotate the Stokes bases such that $P_{B} S_{Y} P_{B}^{\dagger}=S_{Z}=$ $\hat{a}_{H}^{\dagger} \hat{a}_{H}-\hat{a}_{V}^{\dagger} \hat{a}_{V}$. Then we ensure mode $V$ is in the vacuum state, so that we can ignore $\hat{a}_{V}^{\dagger} \hat{a}_{V}$. The final step in the atomic protocol is to perform the measurement of $\hat{q}$, which is achieved by $J_{Y}$.

\section{CONCLUSION}

It is well known that generating large bosonic nonlinearities is exceedingly difficult and so far has primarily been achieved using superconducting quantum optical circuits at microwave frequencies. Finding methods to generate nonlinearities at optical frequencies opens up a wide range of paths for research and applications. In this paper, we presented a protocol that can generate a nonlinearity via a conditional linear operation and measurement with feedback. Using this nonlinearity, we show how to deterministically generate highly nonlinear quantum states of the probe mode which can be very pure. We also show how to engineer the degree of the nonlinearity to depend on unknown parameters, e.g., rotation angle $\theta$. Estimations of this parameter via this nonlinearity can be made with an imprecision which can scale as $\sim 1 / \bar{n}^{3 / 2}$, beating the HL. Perhaps even more useful is the so-called bootstrap method, where we show how to engineer this optical nonlinearity to depend on a wider variety of parameters, e.g., the parameter associated with an unknown displacement, whose estimation is a central task in force metrology. We finally describe a physical setup, using the Faraday interaction of light with atomic ensembles, to implement our protocol. In this case, the unknown rotation angle could be generated by 
a physical magnetic field, thus allowing magnetometry with super-Heisenberg scaling in the sensitivity. At the heart of our protocol is the novel engineering of near-unitary Kraus operations whose random components can almost be completely compensated for. This opens up the ability to perform neardeterministic Schrödinger evolution driven by measurements alone to achieve highly nonlinear and sophisticated quantum dynamics.

\section{ACKNOWLEDGMENTS}

J.T. acknowledges funding from the Australian Research Council Centre of Excellence in Engineered Quantum Systems CE170100009. M.A.R. acknowledges support from Grant No. UNAM-DGAPA-PAPIT IG101421. This paper was partially supported by the U.S. National Science Foundation Grant No. 1630114.
[1] G. J. Milburn and C. A. Holmes, Dissipative Quantum and Classical Liouville Mechanics of the Anharmonic Oscillator, Phys. Rev. Lett. 56, 2237 (1986).

[2] B. Yurke and D. Stoler, Generating Quantum Mechanical Superpositions of Macroscopically Distinguishable States via Amplitude Dispersion, Phys. Rev. Lett. 57, 13 (1986).

[3] W. H. Zurek, Decoherence, einselection, and the quantum origins of the classical, Rev. Mod. Phys. 75, 715 (2003).

[4] A. Luis and L. L. Sánchez-Soto, Breaking the standard quantum limit for interferometric measurements, Opt. Commun. 89, 140 (1992).

[5] W. J. Munro, K. Nemoto, G. J. Milburn, and S. L. Braunstein, Weak-force detection with superposed coherent states, Phys. Rev. A 66, 023819 (2002).

[6] A. Luis, Nonlinear transformations and the Heisenberg limit, Phys. Lett. A 329, 8 (2004).

[7] J. Beltrán and A. Luis, Breaking the Heisenberg limit with inefficient detectors, Phys. Rev. A 72, 045801 (2005).

[8] S. Boixo, S. T. Flammia, C. M. Caves, and J. M. Geremia, Generalized Limits for Single-Parameter Quantum Estimation, Phys. Rev. Lett. 98, 090401 (2007).

[9] S. Boixo, A. Datta, S. T. Flammia, A. Shaji, E. Bagan, and C. M. Caves, Quantum-limited metrology with product states, Phys. Rev. A 77, 012317 (2008).

[10] M. J. Woolley, G. J. Milburn, and C. M. Caves, Nonlinear quantum metrology using coupled nanomechanical resonators, New J. Phys. 10, 125018 (2008).

[11] S. Boixo, A. Datta, M. J. Davis, S. T. Flammia, A. Shaji, and C. M. Caves, Quantum Metrology: Dynamics versus Entanglement, Phys. Rev. Lett. 101, 040403 (2008).

[12] C. Gross, T. Zibold, E. Nicklas, J. Estève, and M. K. Oberthaler, Nonlinear atom interferometer surpasses classical precision limit, Nature (London) 464, 1165 (2010).

[13] A. Rivas and A. Luis, Precision Quantum Metrology and Nonclassicality in Linear and Nonlinear Detection Schemes, Phys. Rev. Lett. 105, 010403 (2010).

[14] M. Napolitano and M. W. Mitchell, Nonlinear metrology with a quantum interface, New J. Phys. 12, 093016 (2010).

[15] M. Napolitano, M. Koschorreck, B. Dubost, N. Behbood, R. J. Sewell, and M. W. Mitchell, Interaction-based quantum metrology showing scaling beyond the Heisenberg limit, Nature (London) 471, 486 (2011).

[16] M. J. W. Hall and H. M. Wiseman, Does Nonlinear Metrology Offer Improved Resolution? Answers from Quantum Information Theory, Phys. Rev. X 2, 041006 (2012).

[17] J. Joo, K. Park, H. Jeong, W. J. Munro, K. Nemoto, and T. P. Spiller, Quantum metrology for nonlinear phase shifts with entangled coherent states, Phys. Rev. A 86, 043828 (2012).
[18] K. Berrada and S. Abdel Khalek, Quantum phase estimation for nonlinear phase shifts with entangled spin coherent states of two modes, Laser Phys. 23, 105201 (2013).

[19] K. Berrada, Quantum metrology with SU $(1,1)$ coherent states in the presence of nonlinear phase shifts, Phys. Rev. A 88, 013817 (2013).

[20] J. Cheng, Quantum metrology for simultaneously estimating the linear and nonlinear phase shifts, Phys. Rev. A 90, 063838 (2014).

[21] A. Luis and A. Rivas, Nonlinear Michelson interferometer for improved quantum metrology, Phys. Rev. A 92, 022104 (2015).

[22] A. Luis, I. Morales, and A. Rivas, Nonlinear fiber gyroscope for quantum metrology, Phys. Rev. A 94, 013830 (2016).

[23] M. Beau and A. del Campo, Nonlinear Quantum Metrology of Many-Body Open Systems, Phys. Rev. Lett. 119, 010403 (2017).

[24] C.-P. Wei and Z.-M. Zhang, Improving the phase sensitivity of a Mach-Zehnder interferometer via a nonlinear phase shifter, J. Mod. Opt. 64, 743 (2017).

[25] J. Flórez, E. Giese, D. Curic, L. Giner, R. W. Boyd, and J. S. Lundeen, The phase sensitivity of a fully quantum three-mode nonlinear interferometer, New J. Phys. 20, 123022 (2018).

[26] X. Nie, J. Huang, Z. Li, W. Zheng, C. Lee, X. Peng, and J. Du, Experimental demonstration of nonlinear quantum metrology with optimal quantum state, Sci. Bull. 63, 469 (2018).

[27] D. V. Tsarev, T. V. Ngo, R.-K. Lee, and A. P. Alodjants, Nonlinear quantum metrology with moving matter-wave solitons, New J. Phys. 21, 083041 (2019).

[28] J.-D. Zhang, Z.-J. Zhang, L.-Z. Cen, J.-Y. Hu, and Y. Zhao, Nonlinear phase estimation: parity measurement approaches the quantum Cramér-Rao bound for coherent states, Phys. Rev. A 99, 022106 (2019).

[29] W. J. Munro, K. Nemoto, and T. P. Spiller, Weak nonlinearities: a new route to optical quantum computation, New J. Phys. 7, 137 (2005).

[30] V. V. Albert, S. O. Mundhada, A. Grimm, S. Touzard, M. H. Devoret, and L. Jiang, Pair-cat codes: autonomous errorcorrection with low-order nonlinearity, Quantum Sci. Technol. 4, 035007 (2019).

[31] A. L. Grimsmo, J. Combes, and B. Q. Baragiola, Quantum Computing with Rotation-Symmetric Bosonic Codes, Phys. Rev. X 10, 011058 (2020).

[32] G. Kirchmair, B. Vlastakis, Z. Leghtas, S. E. Nigg, H. Paik, E. Ginossar, M. Mirrahimi, L. Frunzio, S. M. Girvin, and R. J. Schoelkopf, Observation of quantum state collapse and revival 
due to the single-photon Kerr effect, Nature (London) 495, 205 (2013).

[33] N. C. Menicucci, S. T. Flammia, and P. van Loock, Graphical calculus for Gaussian pure states, Phys. Rev. A 83, 042335 (2011).

[34] D. A. R. Dalvit, R. L. De Matos Filho, and F. Toscano, Quantum metrology at the Heisenberg limit with ion trap motional compass states, New J. Phys. 8, 276 (2006).

[35] R. A. Fisher, Theory of statistical estimation, Math. Proc. Cambridge Philos. Soc. 22, 700 (1925).

[36] C. W. Helstrom, Minimum mean-squared error of estimates in quantum statistics, Phys. Lett. A 25, 101 (1967).

[37] S. L. Braunstein and C. M. Caves, Statistical Distance and the Geometry of Quantum States, Phys. Rev. Lett. 72, 3439 (1994).

[38] S. L. Braunstein, C. M. Caves, and G. J. Milburn, Generalized uncertainty relations: theory, examples, and Lorentz invariance, Ann. Phys. 247, 135 (1996).

[39] V. Giovannetti, S. Lloyd, and L. MacCone, Advances in quantum metrology, Nat. Photonics 5, 222 (2011).

[40] L. Pezzè, A. Smerzi, M. K. Oberthaler, R. Schmied, and P. Treutlein, Quantum metrology with nonclassical states of atomic ensembles, Rev. Mod. Phys. 90, 035005 (2018).

[41] M. G. Paris, Quantum estimation for quantum technology, Int. J. Quantum Inform. 7, 125 (2009).

[42] A. Monras, Optimal phase measurements with pure Gaussian states, Phys. Rev. A 73, 033821 (2006).

[43] R. J. Sewell, M. Napolitano, N. Behbood, G. Colangelo, F. Martin Ciurana, and M. W. Mitchell, Ultrasensitive Atomic Spin Measurements with a Nonlinear Interferometer, Phys. Rev. X 4, 021045 (2014).
[44] J. Combes, C. Ferrie, Z. Jiang, and C. M. Caves, Quantum limits on postselected, probabilistic quantum metrology, Phys. Rev. A 89, 052117 (2014).

[45] F. Albarelli, M. A. C. Rossi, M. G. A. Paris, and M. G. Genoni, Ultimate limits for quantum magnetometry via time-continuous measurements, New J. Phys. 19, 123011 (2017).

[46] Y. Ma, M. Pang, L. Chen, and W. Yang, Improving quantum parameter estimation by monitoring quantum trajectories, Phys. Rev. A 99, 032347 (2019).

[47] F. Toscano, D. A. R. Dalvit, L. Davidovich, and W. H Zurek, Sub-Planck phase-space structures and Heisenberglimited measurements, Phys. Rev. A 73, 023803 (2006).

[48] T. Hillmann, F. Quijandría, G. Johansson, A. Ferraro, S. Gasparinetti, and G. Ferrini, Universal Gate Set for ContinuousVariable Quantum Computation with Microwave Circuits, Phys. Rev. Lett. 125, 160501 (2020).

[49] T. Holstein and H. Primakoff, Field dependence of the intrinsic domain magnetization of a ferromagnet, Phys. Rev. 58, 1098 (1940).

[50] I. H. Deutsch and P. S. Jessen, Quantum control and measurement of atomic spins in polarization spectroscopy, Opt. Commun. 283, 681 (2010).

[51] K. Jacobs, Quantum Measurement Theory and its Applications (Cambridge University Press, Cambridge, 2014).

[52] M. H. Munoz-Arias, P. M. Poggi, P. S. Jessen, and I. H. Deutsch, Simulating Nonlinear Dynamics of Collective Spins via Quantum Measurement and Feedback, Phys. Rev. Lett. 124, 110503 (2020).

Correction: The previously published Figure 2 contained improperly ordered panels and has been replaced. 